\title{
Mythos
}

MYTHOS

Rivista di Storia delle Religioni

$10 \mid 2016$

Varia

\section{Stamatia Dova, Greek Heroes in and out of Hades}

Gabriela Cursaru

\section{OpenEdition}

Journals

Édition électronique

URL : http://journals.openedition.org/mythos/545

DOI : 10.4000/mythos.545

ISSN : 2037-7746

\section{Éditeur}

Salvatore Sciascia Editore

\section{Édition imprimée}

Date de publication : 1 décembre 2016

Pagination : 248-251

ISBN : 978-88-8241-476-4

ISSN : 1972-2516

\section{Référence électronique}

Gabriela Cursaru, "Stamatia Dova, Greek Heroes in and out of Hades », Mythos [En ligne], 10 | 2016, mis en ligne le 24 septembre 2019, consulté le 25 septembre 2020. URL : http://journals.openedition.org/ mythos/545 ; DOl : https://doi.org/10.4000/mythos.545 


\section{Stamatia Dova}

Greek Heroes in and out of Hades

Lexington Books, Lanham, MD 2012, pp. 242, ISBN 9780739144978, \$ 39

Gabriela Cursaru - Université de Montréal - gabriela.cursaru@gmail.com

Le héros grec face à lui-même après sa mort constitue le noyau de ce livre qui, loin de nous plonger dans le royaume des ombres et de décrire les chemins qui y mènent ou le paysage dans lequel évoluent les personnages - les âmes des héros défunts et les vivants qui, descendant dans l'Hadès et leur rendant visite, leur donnent la " dernière " parole -, se concentre sur leur face à face, sur leurs échanges anxieux sur la mort et le destin posthume, mais surtout sur leurs regards rétrospectifs sur la et leur vie héroïque, sur la condition de mortel et la mortalité. On assiste en quelque sorte au déploiement d'une formule iconique conventionnelle où les personnages en dialogue sont présentés de profil au spectateur, mais tout en ayant l'œil de face, ce qui permet qu'un lien se construise avec celui qui regarde : ils sont entre eux, se regardent les uns les autres, échangent des mots - en fait, de véritables soliloques des héros mythiques défunts sur la vie et la mort, sur leur sort funeste, plus précisément sur les manières dont les circonstances de leur mort affectent leur statut posthume (en termes, faute de nóstos, d'honneurs funéraires et civiques, de gloire éternelle et de préservation sociale de la mémoire de leurs nom et exploits, comme formes d'immortalité culturelle et de compensation héroïque censées défier la mort). Ainsi, leur figure héroïque s'éclaircit davantage de même que la notion de kléos gagne en profondeur grâce aux perspectives nouvelles qu'apportent leur regard en arrière et les (ré)évaluations nostalgiques ou ressentimentaires qu'ils font post mortem, les réquisitoires qu'ils dressent, les enseignements qu'ils livrent. Le propos de chacun ne prend sens que dans son rapport avec sa propre histoire, chacun parle de la mort au miroir autant de sa mort que des exploits accomplis de son vivant et en écho au kléos qu'il revendiquait avant que la mort ne lui coupe court toute tentative d'embellir son destin posthume. Situés dans la proximité la plus intime de la mort, les uns d'un côté du seuil de l'Hadès, les autres plongés dans le royaume des ombres et de l'oubli, l'authenticité est mise en avant. Rien d'informel ou de décontracté dans leurs échanges, la démarche de Dova la conduit alors à scruter avec une acuité particulière les propos des interlocuteurs, notamment les monologues des héros défunts.

Les onze essais réunis dans la première partie de l'ouvrage, "Odysseus and the Poetics of Katábasis ", sont consacrés aux conversations qu'Ulysse engage avec les ombres des morts dans le livre XI de l'Odyssée, sans qu'il descende pour autant dans I'Hadès. Elpénor, anti-héros obscur dont la mort prématurée et non héroïque, un parfait contre-exemple du modèle de la "belle mort" sur le champ de bataille, le prive de tout kléos et même des rites funéraires élémentaires, est l'image parfaite de la vie médiocre et vouée à l'échec de tout mortel ordinaire, de l'impossibilité du nóstos et de l'irréversibilité de la mort. Agamemnon, dont le retour, opposé à celui d'Ulysse, est une image négative parfaite du nóstos, car celuici l'a conduit directement à sa mort violente et tragique, se voit ainsi privé de l'idéal héroïque du kalós thánatos, d'une tombe et des honneurs appropriés et, surtout, de kléos (le sien et celui qu'il aura légué à ses descendants). La rencontre entre Ulysse et Achille s'avère être particulièrement 
instructive, car, dernière confrontation discoursive entre les idéaux héroïques incarnés par les deux héros, elle represents the process of redefining kléos in the Odyssey and, more importantly, the effort represented by the Odyssey to redefine kléos in the epic tradition (p. 16). Titulaire incontestable du kléos áphthiton, le héros paradigmatique de l'lliade révèle lors de son face à face avec Ulysse - et en réplique aux louanges extravagantes que celui-ci lui adresse, incluant un makarismós qui recycle la rhétorique réprobatrice d'Agamemnon dans la querelle qui l'opposait à Achille, dans le chant I de l'lliade, v. 287-288, ainsi que Dova le remarque avec justesse - qu'il préférerait la condition d'un pauvre laboureur vivant à celle de prince du royaume des morts. Réévaluation odysséenne saisissante de l'idéal héroïque de la mort au combat et du kléos en tant que fin en soi de tout héros iliadique et, en bonne tradition catabasique, leçon livrée à Ulysse, le survivant par excellence qui est le seul à bénéficier, grâce à ses habiletés et à son endurance, autant de nóstos que du kléos qui en résulte, leçon censée permettre à Ulysse de réévaluer à son tour, à travers les " témoignages " posthumes et le destin funeste des non-survivants, le kléos qu'il réclame et, finalement, la mort qui l'attend: résultante de son nóstos et non pas de ses faits d'armes sur le champ troyen, son kléos ne saurait être que le fruit de sa maturation progressive lors de son voyage de retour.

À telle réévaluation, Ulysse est contraint particulièrement par le refus d'Ajax de s'entretenir avec lui : l'incontestable aristeía de ce héros privé cependant d'une " belle mort " et du kléos spécifique remet légitimement en question le profil héroïque pour le moins controversé d'Ulysse, l'alliage unique de nóstos et kléos auquel il a droit dans l'absence de tout exploit guerrier significatif à Troie. Ulysse évite cependant telle remise en question menaçante pour son kléos (mais, également, pour le concept de kléos dans l'Odyssée en général, un " nóstos-oriented kléos ") par des artifices rhétoriques qui lui sont propres : se heurtant au silence ostentatoire d'Ajax, il remplit cet espace vide de son propre monologue respectueux à l'égard de son ancien compagnon d'armes, en attribuant la responsabilité de sa mort à une instance quasi impersonnelle et arbitraire, aux dieux; ensuite, en maître de son propre itinéraire catabasique, mais aussi de l'économie narrative de la Nékyia, il invite l'interlocuteur suivant à entrer en scène. En prenant habilement le contrôle sur le repli délibéré d'Ajax et sur l'embarras dans lequel le met son silence, Ulysse vainc symboliquement, pour la deuxième fois et toujours avec ses propres armes discoursives bien rusées, ce guerrier iliadique qui seconde Achille en bravoure, mais qui meurt loin du champ de bataille à cause précisément de son manque autodestructif de contrôle. De plus, avec cette pirouette rhétorique, Ulysse remet en question la faillibilité noire du kléos iliadique, que nul autre qu'Ajax incarne le mieux.

Le processus graduel de découverte de soi, processus assimilé à celui de son nóstos, Ulysse l'accomplit au fur et à mesure qu'il se défait de sa persona iliadique, à travers une série de emotional stops (p. 36) - autant de rencontres significatives - et grâce à des agents divins, tous féminins : Circé et Calypso (épisodes patronnés par Hermès et constituant ensemble ce que Dova appelle a long, amorous katábasis), Nausicaa et Arété, Athéna et Inô-Leucothéa. Et ce, sans oublier la rencontre d'Ulysse avec Anticlée dans la Nékyia, mais qui, dans l'économie narrative odysséenne, se situe entre les épisodes de ses visites chez Circé et Calypso : cet entretien avec sa mère défunte, doublé de la consultation nécromantique de Tirésias qui le précède - le télos effectif de la Nékyia - constitue, en effet, l'ouverture vers la vraie katábasis d'Ulysse. C'est à peine à partir de la Nékyia, grâce aux instructions de Tirésias et aux conseils d'Anticlée, que l'odyssée d'Ulysse commence en tant que voyage de retour vers la lumière, vers les siens et chez soi, parcours doublé d'un processus 
" initiatique " de quête et de partage du savoir et de la vérité sur la vie et la mort, sur son propre cheminement avant et après sa mort, au terme de et après son nóstos. Et ce, à travers une longue série d'épreuves durant lesquelles Ulysse confronte la mort et explore les limites de la mortalité, tout en bénéficiant d'une sorte d'immunité contre la peur et l'inconnu ainsi que d'une forme d'" immortalité provisoire "que lui fournissent même sa katábasis réussie (a temporary vaccination against death, $\mathrm{p}$. 76) et la connaissance désormais intime et intériorisée de ce qui sépare la vie et la mort, à laquelle même sa katábasis et les entretiens avec les morts lui ont permis d'accéder. Alors que la katábasis d'Ulysse confirme son choix en tant que seul héros iliadique qui ait droit à un nóstos réussi, c'est son nóstos qui valide non seulement son statut héroïque mais aussi celui d'" initié ", qui forge son kléos et qui lui permet de " renaître ", de renouveler sa vie.

La deuxième partie du livre, "Hadès (and Heroism) Revisited ", utilise l'énigmatique rencontre entre Ulysse et Héraclès (Od. 11.601-626) - en fait, un monologue du vénérable héros, sans réponse de la part d'Ulysse, lequel en tire néanmoins les plus grands bénéfices d'image - comme prélude à une longue discussion sur la katábasis d'Héraclès et son entretien avec Méléagre, qui font l'objet de la cinquième Épinicie de Bacchylide. Leur rencontre n'est pas fortuite, vu les affinités entre les deux héros que Dova scrute avec finesse afin de mieux cerner ce qui les sépare, tout en procédant, en arrière-plan, à une comparaison entre Héraclès et Ulysse. Dans une approche résolument croisée, Dova propose des lectures parallèles de certains épisodes homériques dont Bacchylide se serait servi tout en les adaptant à ses enjeux poétiques et aux valeurs partagées par son auditoire : le duel verbal entre Glaukos et Diomède (II. 6.119-236), modèle selon lequel Méléagre et Héraclès, instead of their intended confrontation, establish family ties between themselves after a significant dialogue of self-discovery (p. 84) ; ou le discours d'Achille dans le livre 9 de l'lliade (225-306) qui, grâce à la lecture attentive de Dova et à la fraîcheur de ses remarques, apporte un éclairage nouveau sur les analogies entre Méléagre et Achille, tout en éclairant leurs portraits grâce à des analyses minutieuses que l'auteure mène de main de maître. Notons ici un seul développement parmi ceux auxquels prêtent les lectures parallèles proposées et qui ouvre une perspective d'interprétation novatrice : ce que Dova appelle inverted katábasis (p. 104), l'attitude de résignation d'Achille devant le sort funeste que les dieux lui ont réservé et de reconciliation avec l'idée de sa mort imminente à Troie, le processus graduel par lequel, tout en suivant cette trajectoire prédéterminée inexorable, il ne consacre le peu de temps qui lui est resté de sa vie que pour donner du sens à sa mort et pour rédefinir son kléos dans ses propres termes.

La discussion autour d'Achille et de l'incompatibilité de sa vision sur l'héroïsme et la mortalité, telle qu'il l'expose dans la Nékyia (notamment Od. 11.488-491), avec les principes et l'agenda éducatifs de la pólis platonicienne (Rép. 3.386c3-7, mais aussi $7.516 c 7-e 3$, où le même monoloque du héros est traité différemment) continue dans la troisième partie de l'ouvrage, "Achilles, Alcestis, and the Poetics of NonKatábasis ", à travers la confrontation du discours d'Achille avec les critiques de Socrate (Rép. 388a-d, 391b2-c6) à l'égard des lamentations excessives du héros après les funérailles de Patrocle et de son refus d'accepter la nécessité et la valeur d'exemple de la "belle mort " de son compagnon (II. 24.3-18). S'y ajoute la confrontation des opinions de Phèdre et de Diotime au sujet de la relation entre Achille et Patrocle (Banq. 178a6-7, 179a5-b3) et du modèle d'héroïsme " féminin " d'Alceste, tel qu'il se dégage de la pièce homonyme d'Euripide et des commentaires platoniciens exposés dans le Banquet par les deux interlocuteurs (179b4-d2 et 208d-e1). Achille et Alceste sapent, chacun à sa manière, le modèle 
traditionnel d'héroïsme et d'attitude devant la mort prôné par la tradition littéraire épique et lyrique et remettent en question la validité, la noblesse et la valeur paradigmatique des notions associées, kléos et kalós thánatos. Par les auto-sacrifices exemplaires par lesquels ils honorent leurs compagnons, Patrocle et Admète, qu'ils soient mus par le pouvoir de l'éros (selon l'opinion de Phèdre) ou par celui d'un désir opportuniste, fallacieux et égocentrique de gloire de leur propre persona (selon Diotime), Achille et sa contrepartie féminine, Alceste, proposent un autre modèle d'héroïsme censé défier la mort et acquérir l'immortalité. L'idéal du kalós thánatos se voit remplacé par celui du kalón érgon (" acte noble "), le kléos par l'éros ou par l'amour de l'immortalité. Quant à la version dramatisée du mythe d'Alceste qu'offre Euripide, version bâtie, selon Dova, sur le modèle folklorique du conflit entre la nouvelle mariée et ses beaux-parents, on assiste à une véritable déconstruction de nombreux motifs traditionnels et des convenances morales et sociales de son époque, à partir de l'inversion des rôles de l'homme et de la femme ou du refus des parents de donner leur vie pour leur fils à cause de leur attachement profond à la vie (philopsychía). Une attention particulière mérite l'analyse que Dova consacre à la katábasis manquée d'Alceste et à la déconstruction de sa mort par Héraclès, Euripide jouant sur le thème de la résurrection et de l'ánodos de l'héroïne en tant que forme d'excellence et sur celui de la possibilité de la traversée dans le double sens de l'espace liminal entre vie et mort, autant que ce passage est ouvert par les dieux et qu'il s'effectue avec l'autorisation divine.

Dova joue avec la matière captivante qu'elle a rassemblée, la répartit en trois parties et une trentaine d'essais distincts suivis de notes très détaillées où des commentaires minutieux, y compris sur les divers développements qu'elle propose à partir des textes soumis à une rigoureuse analyse, enrichissent véritablement la lecture, sans nous éloigner pour autant du fil principal de l'argumentation, tellement le procédé est bien assumé tout au long de l'ouvrage. L'approche est résolument philologique et littéraire : sans schéma préconçu ni esprit d'école, priorité est donnée aux données textuelles. Audelà des analyses des plus profondes et solidement argumentées, on assiste à un parcours foisonnant nourri d'interprétations complexes et bien nuancées, de grande portée psychologique, philosophique et religieuse, et de subtiles hypothèses. On ne regrette que l'absence des conclusions finales dont cet ouvrage, d'une dense concentration thématique, ne saurait qu'en profiter. Cela aurait permis d'ouvrir ou de jeter des ponts vers d'autres pistes de recherche auxquelles le thème de I'héroïsme grec et ses diverses ramifications invitent avec une générosité insoupçonnée, ainsi que le montre, abondamment et de façon très convaincante, même l'ouvrage que Dova nous propose ici. 